\title{
Singularity-free Cosmological Solutions with Non-rotating Perfect Fluids
}

\author{
A. K. Raychaudhuri \\ Relativity and Cosmological Center, Department of Physics, Jadavpur University, Kolkata 700032, India.
}

\begin{abstract}
The paper establishes the result that solutions of the type described in the title of the article are only those that have been already presented in the literature. The procedure adopted in the paper is somewhat novel - while the usual practice is to display an exact solution and then to examine whether it is singularity free, the present paper discovers the conditions which a singularity free solution of the desired type must satistfy. There is no attempt to obtain exact solutions. Simply, the conditions that were ad-hoc introduced in the deduction of singularity free solutions are here shown to follow from the requirement of non-singularity.
\end{abstract}

PACS numbers: 04.20Dw, 04.20Ex, 04.20Jb

\section{INTRODUCTION}

A number of singularity theorems proved in the late sixties of the last century and afterwards led to a belief that a singularity free cosmological solution in general relativity is not possible unless one violates the strong energy condition and/or causality. Here we are not intererested in a critique of these theorems or of the justification of the belief about the non-existence of singularity free solutions. We simply note that when in 1990 Senovilla [1] presented a non-singular solution with a perfect fluid having $\rho=3 p>0$, it appeared to be something exceptional. Shortly afterwards, Ruiz and Senovilla [2] presented a family of non-singular solutions for perfect fluids with or without an equation of state. None of these investigations were motivated by a specific search for non-singular solutions but came as a by-product of an exhaustive investiagtion of $G_{2}$ cosmologies with the following mathematically simplifying assumptions:

(a) The two killing vectors commute and are hyper-surface orthogonal.

(b) The metric tensor components are separable functions i.e., one can write $g_{\mu \nu}=T_{\mu \nu} R_{\mu \nu}$ where $T$ 's and $R$ 's are functions of the time coordinate $t$ and the space coordinate $r$ respectively. The coordinates $t$ and $r$ are respectively the lines tangential to the velocity vector and the space coordinate orthogonal to the spaces spanned by the killing vectors.

(c) All the functions $T_{\mu \nu}$ are expressible as powers of a single function of $t$.

Ruiz and Senovilla did not assume cylindrical symmetry but noted with surprise that all singularity free solutions discovered by them had this symmetry and wondered whether "this type of symmetry could have some relevance to the avoidance of singularity."

Somewhat later, Mars [3] gave up the assumption that the killing vectors are hyper-surface orthogonal and used an "ansatz of separation of variables in comoving coordinates" analogous to the assumption (b) above. However, the condition of separatibilty as usually understood does not fully hold good in his solution. [The exception is $g_{\phi \phi}=r^{2} \cosh (2 a t)+a^{2} r^{4} \cosh ^{-1}(2 a t)$.] Mars's solution had the equation of state $\rho=p$. Ruiz and Senovilla have clearly demonstrated that the only non-singular solutions satisfying their assumptions are given by their equation (58), Mars's work showed that the generalization to nonorthogonal $G_{2}$ adds merely one member to the family of non-singular solutions with non-rotating perfect fluids.

It is not unreasonable to think that these non-singular solutions (discovered, so to say accidentally) are just a subset of measure zero amongst an infinite family of nonsingular solutions - the discovery of the particular ones has been facilitated by their simple mathematical form originating from the very strong assumptions of Ruiz and Senovilla. Such a view would have been confirmed if during the last decade some non-singular solution outside the already discovered family were found. But as nothing like that has happened, it is tempting to nurse the alternative idea that the already discovered solutions exhaust all possible non-singular solutions with non-rotating perfect fluids irrespective of any other consideration. The present investigation was motivated by such an idea and establishes this result by deducing all the assumptions of Ruiz and Senovilla as well as Mars from the condition of non-singularity. But first let us spell out the meaning of different terms used by us:

(i) Non-singularity: It is meant to indicate that none of the physical or geometrical scalars blow up even at infinity. The metric tensor components may blow up (indeed some do blow up in Ruiz-Senovilla and Mars solutions) at spatial or temporal infinity but they must be bounded and continuous in the finite region of space-time. We make no use of the sophisticated idea of geodesic completeness in consideration of singularity. Rather we feel that identifying singularities with incompleteness of null and/or timelike geodesics is in the ultimate analysis 'a piece of opportunism as it allows many theorems to be proved' 河.

(ii) Non-rotating: The fluid flow is assumed to be hydrodynamic so that one can introduce a comoving coordinate system with the velocity vector hyper-surface orthogonal, i.e., $\omega^{\mu}=\epsilon^{\mu \nu \alpha \sigma} v_{\nu} v_{\alpha ; \sigma}=0$. 
(iii) Perfect fluid - The energy stress tensor is of the form $T_{\nu}^{\mu}=(p+\rho) v^{\mu} v_{\nu}-p \delta_{\nu}^{\mu}$ in the usual notation. This means that we are just leaving out viscosity, heat flow etc so that the solution presented by Dadhich et al |5] are outside our purview.

(iv) Cosmological: The energy tensor obeys the strong energy condition - in particular for the perfect fluid we take somewhat stronger constraint $0 \leq p \leq \rho$. The pressure and the density must not vanish anywhere except at infinity. We thus exclude the case of a complete vacuum or a bounded distribution of matter. We shall exclude any discontinuity in $T_{\mu \nu}$ - although that is not inconsistent with absence of singularities, such discontinuities are unusual for simple cosmological models. This will allow us to use a single coordinate system as globally valid.

(v) We further introduce the physical assumption that changes of $p$ and $\rho$ are in the same direction for any change of the coordinates. Such a condition seems natural for any physically acceptable model.

It is easy to see that in the absence of rotation, a nonsingular cosmological solution of the desired type must have both acceleration and expansion non-vanishing. For, in the absence of acceleration, expansion will necessarily be associated with a collapse singularity as in the Friedmann models and the expansion free case is not of interest in cosmology. (They are usually studied as stellar models.)

Dadhich [6] has claimed that instead of all the assumptions of Ruiz and Senovilla, if one, besides our assumptions, assumes that in the comoving coordinate system, the metric tensor is diagonal and separable into functions of time and space coordinates, one is uniquely led to the family discovered by Ruiz and Senovilla. But the additional assumptions of Dadhich are quite strong and leave out Mars's non-diagonal metric. Our treatment is free of these shortcomings and assumes nothing besides the five conditions stated above.

It may be of some interest to note a paper by Senovilla [7]. The Senovilla paper adopts the metric:

$$
\begin{gathered}
d s^{2}=T^{2(1+n)} \Sigma^{2 n(n-1)}\left(-d t^{2}+d r^{2}\right)+T^{2(1+n)} \Sigma^{2 n} \Sigma^{\prime 2} d \phi^{2} \\
+T^{2(1-n)} \Sigma^{2(1-n)} d z^{2}
\end{gathered}
$$

where $T$ is a function of $t$ alone and $\Sigma$ is a function of $r$ alone. This metric satisfies all the conditions used by Ruiz-Senovilla in their deduction of the singularity free perfect fluid solutions, which under the circumstances are shown to be only such solutions. Specifically the assumptions are existence of $G_{2}$, orthogonal separable metric, all time functions in the metric are powers of a single function. Thus any non-singular solution (they are not explicitly displayed) that 7 may imply which differs from that of the Ruiz-Senovilla will either violate the perfect fluid condition (note that Senovilla allows $p_{r} \neq p_{z}$ ) or violates the strong energy condition. (Senovilla himself points out that there are "almost FLRW model" which are singularity free but "violate energy conditions".)

\section{THE COORDINATE SYSTEM AND THE FUNCTIONS $P, \rho, \dot{V}_{\mu}$ AND $\theta$}

The non-rotating condition allows us to write the metric in the form:

$$
d s^{2}=g_{00} d t^{2}+g_{i k} d x^{i} d x^{k} \quad(i, k=1,2,3)
$$

with the fluid velocity components

$$
v^{i}=0, \quad v^{0}=\frac{1}{\sqrt{g_{00}}}
$$

The acceleration vector has the components

$$
\dot{v}_{i}=-\frac{1}{2}\left(\ln g_{00}\right), i \quad ; \dot{v}_{0}=0 \text {. }
$$

Thus the acceleration vector is confined to the three space orthogonal to $v^{\mu}$ and in that space it is a gradient vector. Hence we can align a space-like coordinate ( say $x^{1}=r$ ) along this vector which will be orthogonal to the other three coordinate lines and hence (11) will be reduced to

$$
d s^{2}=g_{00} d t^{2}+g_{11} d r^{2}+g_{a b} d x^{a} d x^{b}
$$

with

$$
\dot{v}_{a}=0, ; \quad(a, b=2,3)
$$

We shall consider the domain of the coordinates to extend from $-\infty$ to $+\infty$. In particular we note that as the tangent to the $r$ coordinate lines is a gradient vector, any $r$-line cannot form a closed loop. Thus $r$ has to run from $-\infty$ to $+\infty$ (or in case the $r$-lines diverge from a point as the radial lines in spherical or cylindrical symmetry, $r$ will run from zero to $\infty$.

One may wonder whether the metric form (4) is globally valid with a single coordinate system. However, we note that our condition (iv) setting our idea of a cosmological solution ensures this global validity. Obviously, because of (5) and (3), $g_{00}$ is a function of $r$ and $t$ only (not of $\left.x^{2}, x^{3}\right)$. From the relation $T_{\nu ; \mu}^{\mu}=0$, we get for perfect fluids,

$$
p,_{i}=-\frac{p+\rho}{2}\left(\ln g_{00}\right),{ }_{i}
$$

Hence $p$ is also a function of $r$ and $t$ only. Writing equation (6) in the form

$$
(p+\rho)=-\frac{2 p,_{i}}{\left(\ln g_{00}\right)_{i}}
$$

one sees that $\rho$ is also a function of $r$ and $t$ only. From the other divergence relation

$$
\frac{1}{\sqrt{g_{00}}} \dot{\rho}=-\frac{p+\rho}{2} \theta
$$

one sees that $\theta$ is also a function of $r$ and $t$ only. Summing up, the scalars $p, \rho, \theta$ and the metric tensor component $g_{00}$ (whose gradient determines the acceleration) are all functions of $r$ and $t$ only. 


\section{THE VANISHING OF SOME FIRST DERIVATIVES}

In two communications [8.9], the present author established that for non-singular, non-rotating cosmological solution, both the space-time average and the space average (defined in a suitable manner), of each of the scalars that appear in the Raychaudhuri equation vanish. These results in turn require that for distributions of perfect fluid which are not bounded, these scalars must vanish both at spatial and temporal infinity. Thus $p, \rho, \sigma, \theta, \dot{v}^{\mu} ; \mu$ all vanish at $r, t \rightarrow \pm \infty$. Consequently the positive definite quantities like $p, \rho, \sigma^{2}, \theta^{2}$ must have at least one maximum both in $r$ and $t$.

Consider now the case of pressure $p$. As it is a function of $r$ and $t$, the maximum in $p$ in $r$ defined by $\frac{\partial p}{\partial r}=0$ and $\frac{\partial^{2} p}{\partial r^{2}}<0$ will be a line in the $r, t$ space. Along this line we shall have,

$$
0=d_{L}\left(\frac{\partial p}{\partial r}\right)=\frac{\partial^{2} p}{\partial r^{2}} d r_{L}+\frac{\partial^{2} p}{\partial r \partial t} d t_{L}
$$

The subscript $L$ indicating that differentials along the line are to be taken. Also as $\frac{\partial^{2} p}{\partial r^{2}}<0$ all along the line, the integral $\int \frac{\partial^{2} p}{\partial r^{2}} d r_{L}$ will diverge if the domain of integration be infinite but $\int \frac{\partial^{2} p}{\partial r^{2}} d r_{L}$ on integration gives $\left(\frac{\partial p}{\partial r}\right)_{L}$ and will be finite for all limits of integration. Thus the only way to satisfy (8) is to have $d r_{L}=0$ and $\frac{\partial^{2} p}{\partial r \partial t}$ also zero at all points on the line. Thus this line is a $t$-line defined by a constant value of $r$.

An exactly similar analysis leads to the conclusion that $\left(\frac{\partial p}{\partial t}\right)=0$ line is a $t$-constant line, i.e., a $r$ coordinate line and over it also $\frac{\partial^{2} p}{\partial r \partial t}$ vanishes. choosing the intersection of these two orthogonal lines as the origin $(r=0, t=0)$ we may sum up as follows:

$$
\begin{gathered}
\frac{\partial p}{\partial r}=0 \text { for } \mathrm{r}=0 \\
\frac{\partial p}{\partial t}=0 \text { for } \mathrm{t}=0 \\
\frac{\partial^{2} p}{\partial r \partial t}=0 \text { if either } \mathrm{r}=0 \text { or } \mathrm{t}=0
\end{gathered}
$$

Exactly similarly,

$$
\begin{gathered}
\frac{\partial \rho}{\partial r}=0 \text { for } \mathrm{r}=\text { constant }=\mathrm{r}_{0} \quad \text { (say) } \\
\frac{\partial \rho}{\partial t}=0 \text { for } \mathrm{t}=\text { constant }=\mathrm{t}_{0} \quad \text { (say) } \\
\frac{\partial^{2} \rho}{\partial r \partial t}=0 \text { if either } \mathrm{r}=\mathrm{r}_{0} \text { or } \mathrm{t}=\mathrm{t}_{0}
\end{gathered}
$$

We have introduced $r_{0}, t_{0}$ to indicte the possibility that the maximum of $p$ may not coincide with the maximum of $\rho$. However, Such a situation will go against our assumption $(\mathrm{v})$. Hence we have $r_{0}=t_{0}=0$.

Next consider the case of $\left(\ln g_{00}\right)$. In view of equation (6), the zero of $\frac{\partial p}{\partial r}$ is also a zero of $\frac{\partial\left(\ln g_{00}\right)}{\partial r}$.

From (6), we get,

$$
\int \frac{\partial p / \partial r}{p}=-\frac{1}{2} \int\left(1+\frac{\rho}{p}\right) \frac{\partial\left(\ln g_{00}\right)}{\partial r}
$$

If the integral extends from $-\infty$ to $+\infty, p \rightarrow 0$, and hence as $\left(1+\frac{\rho}{p}\right)$ is finite and positive everywhere, $g_{00} \rightarrow+\infty$ as $r \rightarrow \pm \infty$. Again, from (7), by a suitable similar integration, $\mid$ det $g_{i k}|\equiv| h \mid \rightarrow+\infty$ as $t \rightarrow \pm \infty$. But $\theta$ and $\dot{v}^{\mu} ; \mu$ vanish at the infinities, consequently $g_{00} \rightarrow+\infty$ also at $t \rightarrow \pm \infty$ and $|h| \rightarrow \infty$ as $r \rightarrow \pm \infty$. Hence both $|h|$ and $g_{00}$ must have minimum with respect to both $r$ and $t$.

That these minina will occur at $r=0$ and $t=0$ again follows from $(\sqrt{6}),(7),(9)$ and $(10)$. A further investigation of the vanishing of $\sigma^{2}$ and $\dot{v}^{\mu} ; \mu$ at the infinities show that $g_{r r}$ and $\gamma$ (the two dimensional metric determinant $=\frac{|h|}{\left|g_{11}\right|}$ ) also blow up at the infinities.

Thus we get

$$
\begin{gathered}
2 \dot{v}^{\mu}=\frac{\partial\left(\ln g_{00}\right)}{\partial r}=0 \text { for } \mathrm{r}=0 \\
\frac{\partial\left(\ln g_{00}\right)}{\partial t}=0 \text { for } \mathrm{t}=0 \\
\frac{\partial^{2}\left(\ln g_{00}\right)}{\partial r \partial t}=0 \text { if either } \mathrm{r}=0 \text { or } \mathrm{t}=0
\end{gathered}
$$

Similarly from (7), we get,

$$
\begin{gathered}
\frac{\partial \ln |h|}{\partial r}=0 \text { for } \mathrm{r}=0 \\
2 \sqrt{g_{00}} \theta=\frac{\partial \ln |h|}{\partial t}=0 \text { for } \mathrm{t}=0 \\
\frac{\partial^{2} \ln h}{\partial r \partial t}=0 \text { if either } \mathrm{r}=0 \text { or } \mathrm{t}=0
\end{gathered}
$$

The pair of orthogonal lines $r=0$ and $t=0$ are the lines of vanishing of first derivatives of $p, \rho, \ln g_{00}, \ln h$. It is easy to see that at no other value of $r$, can $\frac{\partial p}{\partial r}$ vanish. If possible let us suppose that at $r=R(\neq 0), \frac{\partial p}{\partial r}=0$. From our previous analysis, it is clear that non-singularity requires that this cannot be an isolated point but would be the entire $t$ coordinate line at which $r=R$. Also as $r=R$ follows the maximum of $p$ at $r=0, \frac{\partial^{2} p}{\partial r^{2}}$ will benegative or zero over the entire line. Consider now the Raychaudhuri equation,

$$
2 \sigma^{2}+\frac{1}{3} \theta^{2}+\frac{4 \pi}{3}(\rho+3 p)=\dot{v}^{\mu} ;_{\mu}-\dot{\theta}
$$


where

$$
\dot{\theta}=\theta,{ }_{\mu} v^{\mu}=\frac{1}{\sqrt{g_{00}}} \frac{\partial \theta}{\partial t}
$$

and $\dot{v}^{\mu} ;_{\mu}=\frac{1}{\sqrt{-g}}\left(\dot{v}^{1} \sqrt{(-g)}\right)_{1}$.

Now all the terms of the left hand side of $(13)$ are positive and the first term on the right is zero or negative as we have just seen. Hence $\dot{\theta}$ is negative at all points on the line and

$$
\int_{-\infty}^{+\infty} \frac{\partial \theta}{\partial t} d t>0
$$

which contradicts the condition of regularity that $\theta$ is zero at both $t \rightarrow \pm \infty$.

In a similar manner it is easy to see that there is no other zero of $\frac{\partial p}{\partial t}$ except at $t=0$.

\section{EXPRESSIONS OF THE DIFFERENT VARIABLES IN TERMS OF SEPARATED FUNCTIONS}

The results of the last section allow us to express $p$ in the following form

$$
p=\Sigma R_{i} T_{i} Q(r, t)
$$

where $R^{\prime}$ s are linearly independent functions of $r$ having $\frac{\partial R}{\partial r}=0$ at $r=0$ and vanishing at $r \rightarrow \pm \infty$. $T^{\prime}$ s are similarly functions of $t$ alone. We have put in the summation to take care of the possibility that there may be more than one function satisfying the imposed conditions. (Indeed if $f(r)$ is one such function then any positive power of $f(r)$ will satisfy the same condition.) $Q(r, t)$ is any function involving both $r$ and $t$ (if possible). However, as there is no other zero of $\frac{\partial p}{\partial r}$ and $\frac{\partial p}{\partial t}$ except those covered in $R^{\prime} \mathrm{s}$ and $T^{\prime} \mathrm{s}$, the drivarive of $Q(r, t)$ with respect to $r$ and $t$ cannot have any zero. Cosequently, if $Q$ is to be bounded everywhere, it must be a constant and can be absorbed in the $R^{\prime} \mathrm{s}$ and $T^{\prime} \mathrm{s}$. (It is assumed that the summation may be over infinite terms to include all possible $R^{\prime}$ s and $T^{\prime}$ s.)

The functions $R$ and $T$ will be determined by the field equations which are second order partial differential equations - hence there can be at most two linearly independent $R$ functions and two $T$ functions. So finally,

$$
p=R_{p} T_{p}+\bar{R}_{p} \bar{T}_{p}
$$

and similarly

$$
\begin{gathered}
\rho=R_{\rho} T_{\rho}+\bar{R}_{\rho} \bar{T}_{\rho} \\
\ln g_{00}=R_{0} T_{0}+\bar{R}_{0} \bar{T}_{0} \\
\ln h=R_{h} T_{h}+\bar{R}_{h} \bar{T}_{h}+M\left(x^{a}\right)
\end{gathered}
$$

where the unbarred and barred functions with the same subscript are linearly independent.

As yet, we have not proved that $h$ is independent of the coordinates $x^{2}, x^{3}$, hence we have added the term $M\left(x^{a}\right)$ in (14d). No such term is required in the other three expressions as $p, \rho, \ln g_{00}$ have been shown to be functions of $r$ and $t$ only.

Putting in the expressions $(14 a, b, c)$ in equation (6), we get,

$$
R_{p}^{\prime} T_{p}+\bar{R}_{p}^{\prime} \bar{T}_{p}=-\frac{1}{2}\left(R_{p} T_{p}+\bar{R}_{p} \bar{T}_{p}+R_{\rho} T_{\rho}+\bar{R}_{\rho} \bar{T}_{\rho}\right)\left(R_{0}^{\prime} T_{0}+\bar{R}_{0}^{\prime} \bar{T}_{0}\right)
$$

where superscript primes indicate differentiation with respect to $r$.

Using now the non-linear dependence of the barred and unbarred functions, we get from equation (15),

$$
\begin{gathered}
\ln g_{00}=R_{0}+T_{0} \\
\rho=a R_{p} T_{p}+b \bar{R}_{p} \bar{T}_{p} \\
p=R_{p} T_{p}+\bar{R}_{p} \bar{T}_{p}
\end{gathered}
$$

where $a$ and $b$ are constants and $R_{0}, T_{0}$ are different from those in (14c). Also,

$$
\ln \bar{R}_{p}=\frac{1+b}{1+a} \ln R_{p}
$$

and

$$
R_{0}=-\frac{2}{1+a} \ln R_{p}=-\frac{2}{1+b} \ln \bar{R}_{p}
$$

Similarly plugging in (14d) in equation (7), we get,

$$
\begin{gathered}
\ln |h|=R_{h}+T_{h}+M\left(x^{a}\right) \\
\ln \bar{T}_{p}=\frac{1+b}{1-a} \frac{a}{b} \ln T_{p} \\
T_{h}=-\frac{2}{1+a} \ln T_{p}
\end{gathered}
$$

We have already noted that $g_{11}$ and $\gamma$ also blow up at the infinities of $r$ and $t$. Hence they also must be expressible in form similar to the above. Keeping in mind the relation $|h|=|\gamma|\left|g_{11}\right|$, we can write,

$$
\ln \left|g_{11}\right|=R_{1}+T_{1}+N\left(x^{a}\right)
$$

$$
\ln |\gamma|=R_{\gamma}+T_{\gamma}+P\left(x^{a}\right)
$$

Note that we can make transformations $\bar{t}=f(t)$ and $\bar{r}=\phi(r)$ to have

$$
\ln \left|g_{11}\right|-\ln g_{00}=N\left(x^{a}\right)
$$

or, $g_{11} / g_{00}=$ a function of $x^{a}$. 


\section{PROOF OF THE EXISTENCE OF $G_{2}$}

We shall proceed in several steps. The first step is to prove that in the solutions that we are seeking, shear must be non-vanishing.

For this it is necessary to use the field equation

$$
R_{0 i}=-8 \pi\left(T-T_{0 i}\right)
$$

The above equation for a perfect fluid in irrotational motion can be written in the form

$$
\sigma_{i ; \alpha}^{\alpha}-\sigma_{i}^{l} \dot{v}_{l}=\frac{2}{3} \theta,{ }_{i}
$$

In our present coordinate system this becomes

$$
\sigma_{i \mid k}^{k}=\frac{2}{3} \theta,{ }_{i}
$$

where $\mid k$ indicates covariant differentiation with the three space metric. If now $\sigma_{i}^{k}$ vanishes, $\theta$ will be spatially uniform and then either the situation is static $(\theta=0)$ or the solution will have singularities.

If the three non-zero values of shear eigenvalues are $S_{1}, S_{2}$ and $S_{3}$, we get

$$
S_{1}=\left[\frac{1}{2} \frac{\partial}{\partial t}\left(\ln \left|g_{11}\right|-\frac{1}{6} \frac{\partial}{\partial t}(\ln h)\right] \frac{1}{\sqrt{g_{00}}}\right.
$$

i.e., it is a function of $r$ and $t$ only.

In the next step we show that $\sigma^{2}$ and $\dot{v}_{;}^{\mu}$ are independent of the coordinates $x^{a}$. Looking back at equation (13), we see that in view of (16) and (17) all the terms except $2 \sigma^{2}$ and $\dot{v} ; \mu$ are functions of $r$ and $t$ only. Hence we must have,

$$
\left(2 \sigma^{2}\right)_{a}=\left(\dot{v} ; ;_{\mu}^{\mu}\right),_{a}
$$

But

$$
\begin{gathered}
\dot{v}_{\mu}^{\mu}=\frac{1}{\sqrt{-g}}\left(\dot{v}_{1} g^{11} \sqrt{-g}\right)_{, 1} \\
=-\frac{e^{N / 2}}{g_{00}}\left[\left(\ln g_{00}\right)_{, 11}+\frac{1}{2}\left[\left(\ln g_{00}\right)_{, 1}(\ln \gamma)_{, 1}\right]\right.
\end{gathered}
$$

Excepting $e^{N / 2}, g_{00}$ and the term within the braces are functions of $r$ and $t$ only and these have no zero for finite values of $r$ and $t$. Thus,

$\left(2 \sigma^{2}\right)_{a}=\left(e^{N / 2}\right)_{a} \times$ a non - vanishing expression

Consequently, if $N$ is not a constant $\left(2 \sigma^{2}\right)_{a}$ or $\left(\dot{v} ;_{\mu}^{\mu},,_{a}\right.$ will have no zero in the finite region of $r$ and $t$. But as $\sigma^{2}$ vanishes at $t=0$ for all values of $r$ and $x^{a}$ and $\left(2 \sigma^{2}\right)_{, a}$ or $\left(\dot{v} ; \mu_{\mu}^{\mu}, a\right.$ also vanish there, and it follows that $N$ is a constant and hence

$$
S_{1, a}=S_{2, a}=S_{3, a}=0
$$

$$
g_{11, a}=0
$$

We now come to the last step of our proof of existence of $G_{2}$. For this we introduce three orthonormal vectors $\lambda_{1 \mid}^{i}, \lambda_{2 \mid}^{i}, \lambda_{3 \mid}^{i}$, the three unit eigenvectors of the shear tensor. Using the following formulas given in Eisenhart's book 10

$$
\begin{gathered}
\sigma_{i j ; k} \lambda_{h \mid}^{i} \lambda_{l \mid}^{j} \lambda_{m \mid}^{k}=\left(S_{h}-S_{l}\right) \gamma_{h l m} \quad(h \neq l) \\
\sigma_{i j ; k} \lambda_{h \mid}^{i} \lambda_{h \mid}^{j} \lambda_{l \mid}^{k}=\lambda_{l \mid}^{k} \frac{\partial S_{h}}{\partial x^{k}}
\end{gathered}
$$

we get from (21),

$$
\begin{aligned}
& \frac{2}{3} \theta,_{1}=\left[S_{1,1}+\frac{1}{\lambda_{\mid 1}^{1}}\left(S_{1}-S_{2}\right) \gamma_{122}+\frac{1}{\lambda_{\mid 1}^{1}}\left(S_{1}-S_{3}\right) \gamma_{133}\right] \\
& 0=\left[S_{2,2}+\frac{1}{\lambda_{\mid 2}^{2}}\left(S_{2}-S_{3}\right) \gamma_{233}+\frac{1}{\lambda_{\mid 2}^{2}}\left(S_{2}-S_{1}\right) \gamma_{211}\right] \\
& 0=\left[S_{3,3}+\frac{1}{\lambda_{\mid 3}^{3}}\left(S_{3}-S_{2}\right) \gamma_{322}+\frac{1}{\lambda_{\mid 3}^{3}}\left(S_{3}-S_{1}\right) \gamma_{311}\right]
\end{aligned}
$$

where $\gamma^{\prime}$ s are Ricci relation coefficients. As $g_{11, a}=0, \lambda_{1 \mid}^{i}$ is geodetic in the three space. Hence $\gamma_{211}=\gamma_{311}=0$. Now from (25) and (26) as $S_{i, a}=0$ we get, if $S_{2} \neq S_{3}$

$$
\gamma_{233}=\gamma_{322}=0
$$

If $S_{2}=S_{3}$, then the existence of $G_{2}$ can be readily proved but as will be apparent from our later discussion, this case will lead to the vanishing of shear and hence to singular solutions. From (27), owing to the antisymmetry $\gamma_{a b c}=-\gamma_{b a c}$, it follows that all the $\gamma^{\prime} s$ involving the subscripts 2 and 3 vanish and consequently the two spaces spanned by $x^{2}$ and $x^{3}$ are all flat; i.e., we may choose coordinates such that $g_{a b, c}=0$. Thus all the metric tensor components are independent of $x^{a}$ - the space time admits translations along $x^{2}$ and $x^{3}$.

\section{CYLINDRICAL SYMMETRY}

From the field equations, one can deduce in case of irrotational motion of a perfect fluid (cf. Ellis [11]).

$$
{ }^{*} R=-\frac{2}{3} \theta^{2}+2 \sigma^{2}+16 \pi \rho
$$

where ${ }^{*} R$ is the Ricci scalar for the three space $t=$ const. Now by direct calculation,

$$
{ }^{*} R_{1}^{1}=-\frac{1}{\sqrt{-g_{11}}} \frac{\partial \Theta}{\partial r}-\frac{1}{2} \Theta^{2}-2 \Sigma^{2}
$$


where $\Theta$ and $\Sigma$ are the 'expansion' and 'shear scalar' for the unit space-like vector $\xi^{i}=\frac{1}{\sqrt{-g_{11}}} \delta_{1}^{i}$, i.e.,

$$
\begin{gathered}
\Theta=\xi_{\mid i}^{i}=\frac{1}{\sqrt{-g_{11}}}(\ln \sqrt{\gamma})_{, 1} \\
2 \Sigma^{2}=\Sigma_{i k} \Sigma^{i k} \\
\Sigma_{i k}=\frac{1}{2}\left(\xi_{i \mid k}+\left(\xi_{k \mid i}\right)-\frac{1}{2}\left(g_{i k}+\left(\xi_{i} \xi_{k}\right) \xi_{\mid m}^{m}\right.\right.
\end{gathered}
$$

where $\mid i$ indicates covariant differentiation with the three spaces metric. The reader may note the similarity between equation (29) and the Raychaudhuri equation in the 4-dimensional space time. Again with $G_{2}$,

$$
{ }^{*} R={ }^{*} R_{1}^{1}-\frac{1}{\sqrt{-g_{11}}} \frac{\partial \Theta}{\partial r}-\Theta^{2}
$$

Combining (28), (29) and (30), one gets,

$$
-\frac{2}{\sqrt{-g_{11}}} \frac{\partial \Theta}{\partial r}-\frac{3}{2} \Theta^{2}=-\frac{2}{3} \theta^{2}+2 \sigma^{2}+16 \pi \rho+2 \Sigma^{2}
$$

For the three space at $t=0, \theta^{2}=\sigma^{2}=0$ and we get from (31)

$$
-\left(\frac{2}{\sqrt{-g_{11}}} \frac{\partial \Theta}{\partial r}+\frac{3}{2} \Theta^{2}\right)>0
$$

The inequality (32) shows that $\Theta$ would blow up leading to the collapse of the two-space (i.e., $\gamma=0$ ). This will not mean a singularity iff $x^{2}$ or $x^{3}$ or both be angle coordinate so that $\gamma$ will naturally vanish at $r=0$. However both cannot be angle coordinates for then $\Sigma^{2}$ would vanish at $r=0$ and (31) would make $\rho$ blow up at that point. With one only of $x^{2}, x^{3}$ being angle coordinate both the left hand side as well as $\Sigma^{2}$ would blow up leaving a finite difference for $\rho$.

Thus one of the translations is along an angle coordinate indicating that we have cylindrical symmetry.

\section{RELATION BETWEEN DIFFERENT TIME FUNCTIONS}

Let us identify $x^{2}$ as the angle coordinate. Then the condition of elementary flatness gives $S_{1}=S_{2}$. Plugging in this result in (24), we get

$$
S_{1,1}+3 \frac{S_{1}}{2}\left(\ln g_{33}\right)_{1}=\frac{2}{3} \theta, 1
$$

or

$$
\frac{1}{2} S_{1}\left(\ln g_{00}\right)_{, 1}-3 \frac{S_{1}}{2}\left(\ln g_{33}\right)_{, 1}=\frac{1}{3} \theta\left(\ln g_{00}\right)_{1}
$$

so that

$$
\frac{\left(\ln g_{33}\right)_{1}}{\left(\ln g_{00}\right)_{1}}=\frac{2}{3} \frac{\left.\frac{\left(S_{1}\right.}{2}-\frac{\theta}{3}\right)}{S_{1}}
$$

From what we have proved it is clear that the left hand side of (33) is a function of $r$ alone while the right hand side a function of $t$ only. Hence both sides must be constant. So that we have

$$
S_{2}=S_{1}=n \theta ; \quad S_{3}=-2 n \theta
$$

where $n$ is a constant. Equation (34) leads to the desired result that $T_{1}=T_{2}=T_{0}$, and $T_{h}$ are expressible as powers of a single time function. Equations (17b) and (17c) then show that $T_{p}$ and $\bar{T}_{p}$ are also of the same type. Hence we have deduced the assumption (c) of Ruiz and Senovilla.

At this stage it is clear that we have been able to deduce all the assumptions made by Ruiz and Senovilla except the hypersurface orthonormality of the killing vectors. This is as it should be for Mars has shown that nonsingular solutions do exist where the killing vectors are not hypersurface orthogonal. Also, our demonstration of separatibility stops at that of $\gamma$ and does not include the non-diagonal $g_{a b}$. This again is consistent with Mars's finding.

Thus we may claim to have achieved our objective namely that with our conditions (i) to (v), the already known solutions given by Ruiz-Senovilla and Mars are the only possible non-singular solutions.

\section{CONCLUDING REMARKS}

It is tempting to ask whether the present work can be extended to more general cases, i.e., whether one can establish specific conditions about non-singular cosmological solutions when one or more of the conditions of irrotational motion, perfect fluidity and $\frac{\partial p}{\partial \rho}>0$ is given up. Already there exist quite a number of imperfect fluid non-singular solutions but they vary widely in their characteristics. Thus besides cylindrically symmetric solutions, there are also spherically symmetric solutions 12 and even an oscillatory solution 113. In fact for imperfect fluids, the divergence relations $T_{\mu ; \nu}^{\nu}=0$ written out explicitly are much more complicated than (6) and (7) and do not seem amenable for simple general analysis. again if rotation be present, non-diagonal terms $g_{0 i}$ appear in the metric tensor and further calculation becomes much involved. So far as the condition $\frac{\partial p}{\partial \rho}>0$ is concerned, the present author believes that it can be done away with although right now he is not in a position to show that.

Acknowledgements:

The author's thanks are due to the members of the Relativity and Cosmology Center, Jadavpur University and N. Dadhich of Inter University center for Astronomy and Astrophysics, Pune (India), for helpful discussions. 
[1] J. M. M. Senovilla, Phys. Rev. Lett. 64 ,2219 (1990).

[2] E. Ruiz and J. M. M. Senovilla, Phys. Rev. D45, 1995 (1992).

[3] M. Mars, Phys. Rev. D51, R3989 (1995).

[4] J. Earman,The expanding World of General Relativity, Einstein studies 7 ed. H. Goenner et al, Boston, Barkhauser Verlag (1998).

[5] N. Dadhich, L. K. Patel and R.Tikekar, Pramana - Journal of Physics, 44, 303 (1995).

[6] Inhomogeneous Cosmological Models, eds. A. Molina and J. M. M. Senovilla, World Scientific, Singapore (1999), Pp 63-74.
[7] J. M. M. Senovilla, Phys. Rev. D 53, 1799 (1996).

[8] A. K. Raychaudhuri, Phys. Rev. Lett. 80, 654 (1998).

[9] A. K. Raychaudhuri, Mod. Phys. Lett A15, 391 (2000).

[10] L. P. Eisenhart, Riemannian Geometry, Princeton university Press, Princeton (1950).

[11] General Relativity and Cosmology, Proceedings of the International School of Physics, 'Enrico Fermi' Course XLVII, ed. R. K. Sachs, Academic Press (1991)

[12] N. Dadhich, J. Astrophys. Astro 18, 343 (1997).

[13] N. Dadhich and A. K. Raychaudhuri, Mod. Phys. Lett. A14, 2135 (1999). 